\title{
Functional significance of the platelet immune receptors GPVI and CLEC-2
}

\author{
Julie Rayes ${ }^{1}$, Steve P. Watson, ${ }^{1,2}$ and Bernhard Nieswandt ${ }^{3}$ \\ IInstitute of Cardiovascular Sciences, College of Medical and Dental Sciences, University of Birmingham, Birmingham, United Kingdom. ${ }^{2}$ Centre of Membrane Proteins and Receptors (COMPARE), \\ Universities of Birmingham and Nottingham, United Kingdom. ${ }^{3}$ nstitute of Experimental Biomedicine, University Hospital and Rudolf Virchow Center, University of Würzburg, Würzburg, Germany.
}

\begin{abstract}
Although platelets are best known for their role in hemostasis, they are also crucial in development, host defense, inflammation, and tissue repair. Many of these roles are regulated by the immune-like receptors glycoprotein VI (CPVI) and C-type lectin receptor 2 (CLEC-2), which signal through an immunoreceptor tyrosine-based activation motif (ITAM). GPVI is activated by collagen in the subendothelial matrix, by fibrin and fibrinogen in the thrombus, and by a remarkable number of other ligands. CLEC-2 is activated by the transmembrane protein podoplanin, which is found outside of the vasculature and is upregulated in development, inflammation, and cancer, but there is also evidence for additional ligands. In this Review, we discuss the physiological and pathological roles of CLEC-2 and CPVI and their potential as targets in thrombosis and thromboinflammatory disorders (i.e., disorders in which inflammation plays a critical role in the ensuing thrombosis) relative to current antiplatelet drugs.
\end{abstract}

\section{Introduction}

According to the classical paradigm, platelets' physiological and pathophysiological role is in hemostasis and thrombosis. In the last few years, platelets have been shown to regulate a much wider range of physiological processes, including development, host defense, and tissue repair, and related pathological consequences such as cancer metastasis, autoimmunity, and chronic inflammation. The beneficial or detrimental contribution of platelets to many of these pathways is distinct from the mechanisms that underlie hemostasis and thrombosis, with the receptors glycoprotein VI (GPVI) and C-type lectin-like receptor 2 (CLEC-2) emerging as key players. In this Review, we critically discuss the roles of GPVI and CLEC-2 in platelet regulation and their potential as targets in both hemostatic and non-hemostatic disorders, including chronic inflammatory diseases and cancer.

\section{GPVI}

GPVI is a member of the Ig superfamily of receptors with two Ig domains (D1, D2), a mucin-like stalk rich in $O$-glycosylation sites, and a cytoplasmic tail containing calmodulin- and Src kinasebinding sites $(1,2)$. The expression and signaling of GPVI depend on its association with dimeric Fc receptor $\gamma$-chain $(\mathrm{FcR} \gamma)$, which has an immunoreceptor tyrosine-based activation motif (ITAM) that contains two YxxL sequences. Activation of GPVI leads to Src kinase-dependent phosphorylation of the two conserved tyrosines, binding of the tandem SH2 domains of Syk, and initiation of a signaling cascade that activates phospholipase $\mathrm{C} \gamma 2$ (Figure 1A). GPVI is exclusively expressed in megakaryocytes and platelets, with 3000-4000 copies per human platelet $(1,3)$. The gene encoding GPVI, GP6, is located at 19q13.4 in the human genome (4).

Conflict of interest: The authors have declared that no conflict of interest exists. Reference information: / Clin Invest. 2019;129(1):12-23.

https://doi.org/10.1172/JCI122955.
GPVI-collagen interaction. GPVI is the major signaling receptor for collagen. Collagen binds to the D1 domain in GPVI through a glycine-proline-hydroxyproline (GPO) sequence, with helical peptides based on this sequence, known as collagen-related peptides (CRPs), mimicking collagen-induced platelet activation. Collagen binds to dimeric but not to monomeric GPVI with submicromolar affinity $(5,6)$; crystallization studies suggest that dimerization occurs through the D2 domain (7). Several antibodies and phage proteins bind selectively to recombinant dimeric GPVI but give different estimates of the level of the dimer on resting platelets (2\%-29\% of total protein), making it unclear what is being recognized. These studies all report an increase in dimeric GPVI upon platelet activation $(8,9)$, and this may reinforce collagen signaling. The immobilization of platelets on collagen promotes higherorder clustering of GPVI, further increasing signal strength (10).

GPVI-deficient platelets. The first-described GPVI-deficient patients presented with an autoimmune thrombocytopenic purpura resulting from antibodies to GPVI (11) and bleeding due to the combination of antibody-induced loss of GPVI and thrombocytopenia. Several unrelated families in Chile have an insertion that generates a stop codon prior to the transmembrane domain (12). All of the index case have a normal platelet count and a very mild bleeding diathesis.

Mice with a constitutive deletion in GPVI are born at a Mendelian ratio, have a normal platelet count, and are indistinguishable from littermates (13). They exhibit a mild increase in bleeding times following tail incision, although the limitations of this assay should be acknowledged. Treatment with the mAb JAQ1 induces a sustained loss of GPVI in vivo that is preceded by transient thrombocytopenia $(14,15)$. The loss of GPVI results from binding of the Fc region of the antibody to Fc $\gamma$ RIIB on liver sinusoidal endothelial cells (16). A similar mechanism may underlie loss of GPVI in patients exposed to circulating antibodies (17).

ITAM receptor activation or exposure to high shear leads to shedding of GPVI and glycoprotein 1b (GPIb) close to the trans- 
A

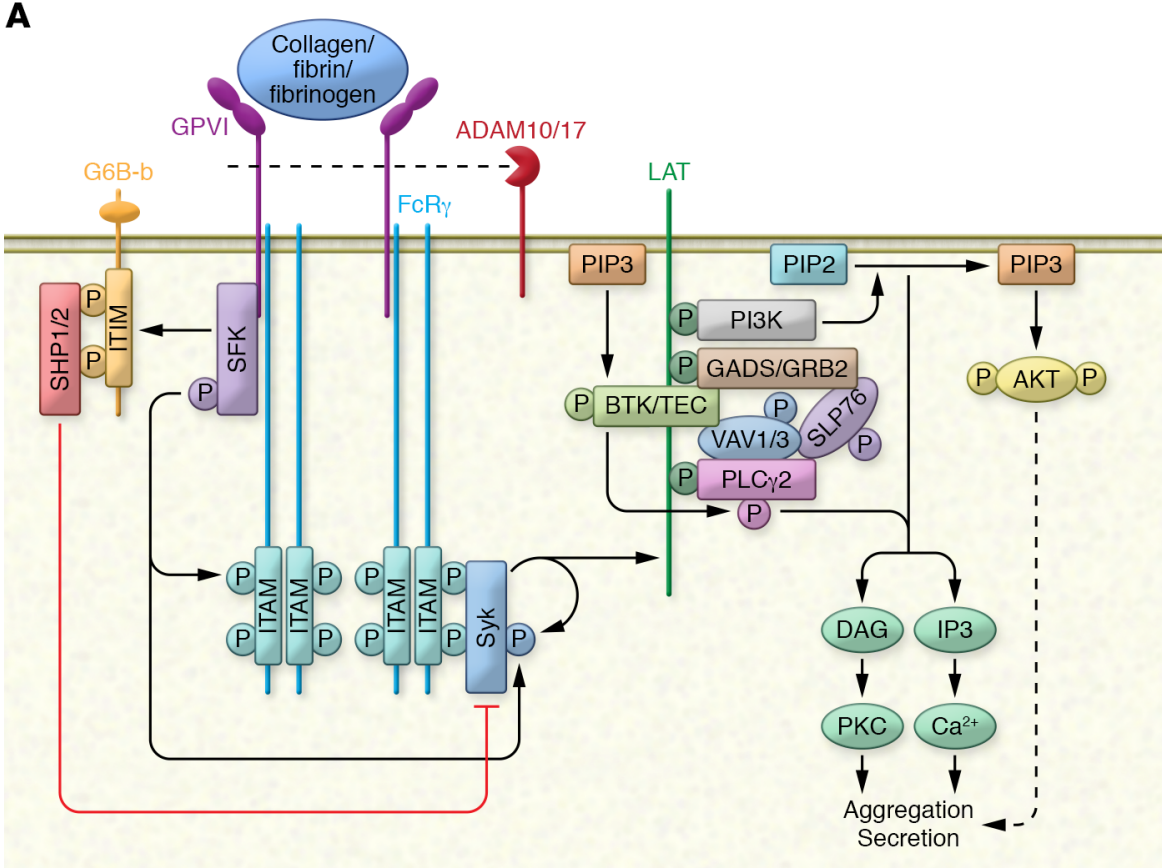

B

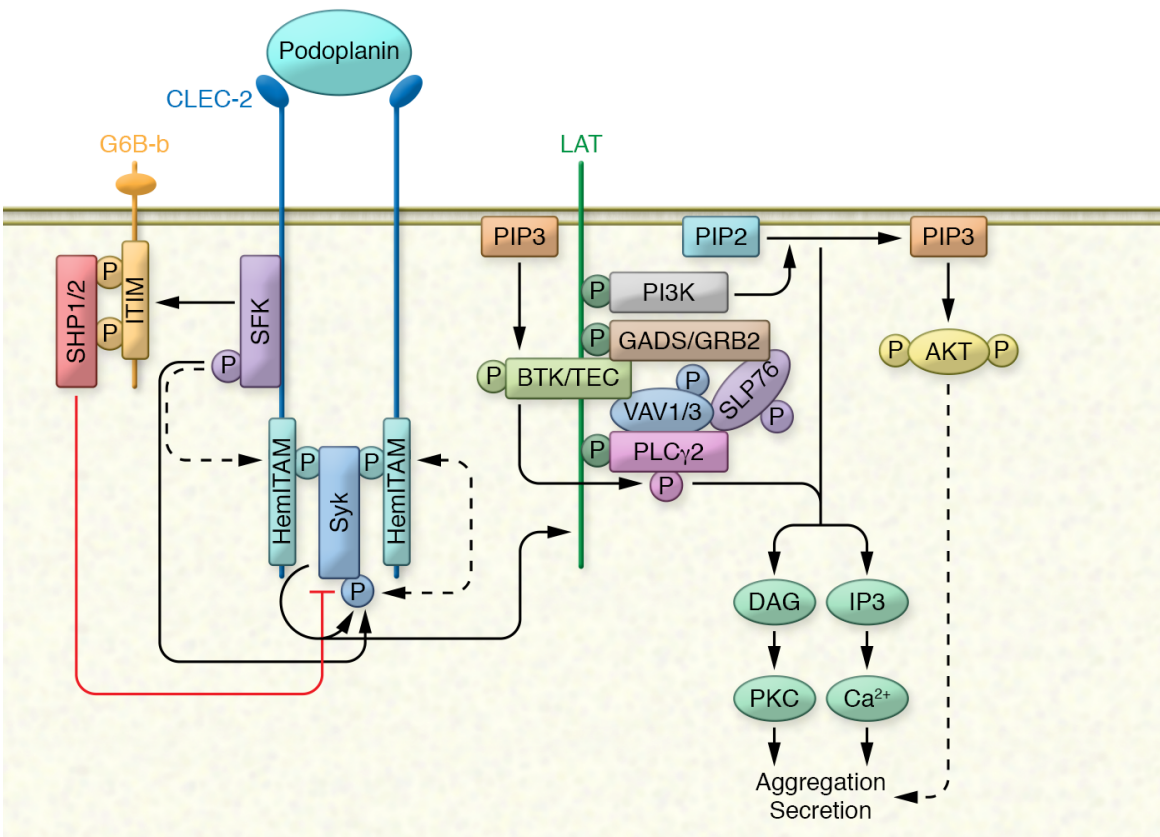

Figure 1. ITAM receptors activate platelets via a (hem)ITAM phosphotyrosine signaling cascade. (A) GPVI activation initiates Src family kinasemediated (SFK-mediated) phosphorylation of two conserved tyrosines in the associated FcR $\gamma$ ITAM and allows the recruitment and docking of Syk's SH2 domain. GPVI signaling is regulated through shedding by ADAM10/17, with G6B-b preventing shedding in circulating platelets via the tyrosine phosphatases SHP-1 and SHP-2. (B) CLEC-2 activation induces phosphorylation on the hem(ITAM), allowing binding of Syk. ( $A$ and B) Once recruited, Syk undergoes a series of autoand trans-phosphorylations by SFK, leading to recruitment and phosphorylation of the adaptor protein LAT and recruitment of this pathway's effectors, such as GRB2, GADS, SLP76, VAV1/3, PIP3, and BTK/TEC. The signaling cascade culminates in the phosphorylation and activation of PLC $\gamma 2$, which binds to phosphorylated LAT and PIP3 on the plasma membrane. PIP3 also recruits BTK and TEC, which phosphorylate PLC $\gamma 2$. Activation of PLC $\gamma 2$ induces formation of the secondary messengers inositol 1,4,5-trisphosphate (IP3) and 1,2-diacylglycerol (DAG), resulting in the mobilization of intracellular $\mathrm{Ca}^{2+}$ stores and activation of PKC, respectively. These changes result in the secretion of intracellular $\alpha$-granules and dense granules and inside-out activation of platelet integrins, including $\alpha_{\mu b} \beta_{3}$, leading to platelet aggregation. CLEC-2 signaling in human platelets depends on actin polymerization and the secondary mediators, ADP and TxA ${ }_{2}$. membrane domain in vitro and in vivo by the metalloproteinases a disintegrin and metalloproteinase domain-containing protein 10 (ADAM10) and ADAM17 $(18,19)$. We speculate that the physiological role of shedding is to limit platelet activation in intact vessels, notably under high shear. The platelet-specific immunoreceptor tyrosine-based inhibition motif (ITIM) receptor G6b-B inhibits shedding of GPVI and GPIb, with genetic deletion of G6b-B resulting in a severe thrombocytopenia and loss of GPVI and GPIb in mice and human (20-22). The GPVI ectodomain (soluble GPVI) is elevated in thrombosis, trauma, and inflammatory conditions, including rheumatoid arthritis and acute ischemic stroke, serving as a specific biomarker of platelet activation $(19,23-26)$.
Additional ligands for GPVI. A remarkable number of ligands for GPVI have been reported, although the majority have been described in a single study, and their significance remains uncertain. These include laminin, which signals through a GPVI- and integrin $\alpha_{6} \beta_{1}$-dependent pathway $(27,28)$; fibronectin and vitronectin, which support adhesion to intact atherosclerotic endothelium and to activated endothelial cells, respectively $(29,30)$; the membrane protein EMMPRIN (CD147, basigin), which supports recruitment of monocytes to immobilized platelets (31); adiponectin (32); and amyloid A 340 peptide (33).

More recently, GPVI was reported to support thrombus growth and stabilization through binding to fibrin and fibrinogen 
(34-37), although interestingly, fibrinogen only activates human GPVI (37). There are contrasting reports on whether the binding to monomeric or dimeric GPVI occurs, possibly due to use of different forms of recombinant or shed GPVI $(35,36,38,39)$. The binding site for fibrin(ogen) resides in the D-region, with D-dimer inhibiting platelet activation by collagen at concentrations that lie at the upper end of those reached in vivo $(36,40)$. The buildup of a fibrin monolayer in a microfluidic model of human venous valve disease was shown to initiate thrombus formation through a pathway that is inhibited by D-dimer (41).

A wide range of exogenous toxins and synthetic ligands activate GPVI, including snake venom toxins (e.g., convulxin), diesel exhaust particles, small peptides, polysulfated sugars, and phosphorothioate antisense oligonucleotides $(42,43)$. The structures of many of the synthetic ligands suggest that these interactions are largely electrostatic, with the multivalent nature of the ligands leading to receptor clustering. Several of these ligands bind to other receptors, and this may facilitate activation of GPVI.

\section{CLEC-2}

CLEC-2 is a type II transmembrane protein containing an extracellular lectin-like recognition domain that lacks its $\mathrm{Ca}^{2+}$ binding site and a short cytosolic tail harboring a single YxxL sequence termed hemITAM $(44,45)$. CLEC-2 is expressed as a dimer (46) and signals through a pathway similar to that of GPVI, with the tandem SH2 domains of Syk bridging two phosphorylated receptors (Figure 1B). CLEC-2 is expressed on platelets and megakaryocytes, and at low levels on inflammatory dendritic cells and CD $11 b^{\text {hi }} \mathrm{Gr}-1^{\text {hi }}$ myeloid cells (47). The reported expression of CLEC-2 on other cells is explained by off-target binding of mAb 17D9 (47). Human and mouse platelets reportedly express $2000-4000$ and $\sim 40,000$ copies of CLEC-2, respectively $(23,48,49)$. The gene encoding CLEC-2, CLEC1B, is located at 12p13.31 in the human genome.

CLEC-2/podoplanin interaction. Podoplanin is a type I single transmembrane protein and the only established endogenous ligand for CLEC-2. Podoplanin is widely expressed outside the vasculature, including on kidney podocytes, lymphatic endothelial cells, choroid plexus, and lung epithelial cells, and is upregulated in many cancers and in inflammation on macrophages, Th17 T cells, and fibroblasts (50).

The extracellular domain of podoplanin contains repeat regions known as platelet aggregation-stimulating (PLAG) domains, an extensively $O$-glycosylated stalk, and a short cytoplasmic tail that binds to the ERM family of cytoskeletal-binding proteins that regulates cell motility (51-54). Crystallization studies have revealed an electrostatic interaction between a conserved EDXXXT/S sequence in PLAG2/3 and four arginines in CLEC-2 (55). The non-glycosylated snake venom rhodocytin binds to the same region in CLEC-2 (55).

CLEC-2- and podoplanin-deficient mice. Mice with a constitutive loss of CLEC-2 or podoplanin are characterized by blood-filled lymphatic vessels in mid-gestation and near $100 \%$ perinatal mortality due to a failure in lung inflation. It was recently shown that the binding of CLEC-2 to podoplanin on lymphatic endothelial cells is required for lung development and that this is involved in the differentiation of lung mesothelial cells into smooth muscle actinpositive alveolar duct myofibroblasts in a TGF- $\beta$-dependent man- ner (56). The degree of blood-lymphatic mixing is reduced in mice with a targeted deletion of CLEC-2 in the megakaryocyte/platelet lineage (PF4-Cre.Clec-1 $b^{f / f f}$ ), and the mice are viable, albeit with a $30 \%$ reduction in platelet count. The reduction in severity may be due to the presence of CLEC-2 on another cell type or because deletion in the megakaryocytic lineage is delayed $(57,58)$. Radiation chimeric mice reconstituted with $\mathrm{Clec}^{-1} b^{-/}$fetal liver cells have a normal platelet count but develop blood-lymphatic mixing in the intestine and exhibit signs of severe malnutrition within the first 3 months $(46,58)$, most likely due to impaired uptake of fat in the intestine. Postnatal deletion of platelet CLEC-2 using a tamoxifeninducible model does not induce blood-lymphatic mixing, thrombocytopenia, or signs of malnutrition, suggesting that CLEC-2 is not required for maintenance of the lymphatics after development (59). Patients lacking CLEC-2 have not been described.

Treatment with the CLEC-2 mAb INU1 achieves sustained depletion of CLEC-2 from mouse platelets in vivo (60) due to internalization and intracellular degradation (61), and severe but transient thrombocytopenia. CLEC-2, however, is not shed from activated platelets, and only very low levels of the CLEC-2 ectodomain are present in blood (62). CLEC-2-positive microvesicles are elevated in the plasma of patients with rheumatoid arthritis (23).

Additional ligands for CLEC-2. Increased tail bleeding times and reduced thrombus formation have been reported in some but not all studies in CLEC-2-deficient mouse models, suggesting the presence of an additional ligand in the vasculature $(46,57,60,63)$. A reduction in thrombus formation is seen in mice expressing a signaling-dead mutant of CLEC-2 only in the presence of a blocking $\mathrm{F}(\mathrm{ab})$ of $\mathrm{mAb}$ INU1, suggesting that CLEC-2 functions as an adhesion receptor (64). The endogenous ligand could be CLEC-2 itself, as it has been reported to undergo homophilic binding with submicromolar affinity (63).

A variety of exogenous ligands activate CLEC-2, including the snake venom toxin rhodocytin, diesel particles, and the sulfated polysaccharides dextran sulfate and fucoidan $(42,65)$. The dependence on sulfation in the polysaccharides may reflect binding to the conserved arginines in CLEC-2 that support its interaction with podoplanin (55).

\section{Hemostasis, thrombosis, and thrombo- inflammation}

Integrin $\alpha_{I I} \beta_{3}$-dependent hemostasis. In arteries and arterioles, hemostasis is achieved through rapid activation of platelets by subendothelial matrix proteins and soluble agonists; this leads to inside-out activation of integrin $\alpha_{\mathrm{IIb}} \beta_{3}$ and a thrombus composed of a densely packed fibrin-rich core and a looser outer shell of platelets, with diffusion of soluble ligands from the core determining the size of the shell (Figure 2A) (66). GPVI was originally presented as a key player in platelet aggregation in in vitro flow models over immobilized collagen, but this is not reflected in vivo due to redundancy with other ligands in the vessel wall, including thrombin (which is generated following exposure of tissue factor) and thromboxanes (67-69). The interaction of fibrin and fibrinogen with GPVI has been proposed to play a role in thrombus assembly and stabilization, and may explain the increase in embolization in GPVI-deficient mice (57).

In comparison, CLEC-2 has a minor-to-negligible role in hemostasis, as shown by in vitro flow studies on collagen and by 
A Classical hemostasis $\left(\alpha_{111} \beta_{3}\right.$-dependent)
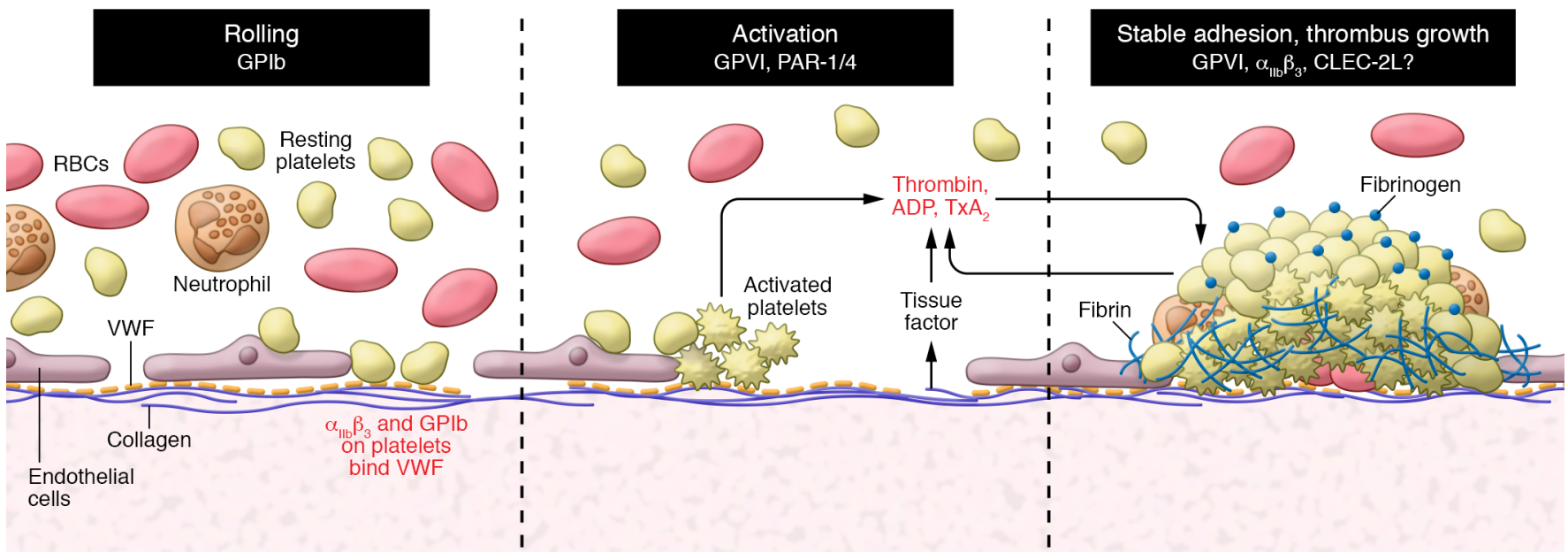

B Inflammatory hemostasis $\left(\alpha_{11 \mathrm{~b}} \beta_{3}\right.$-independent)

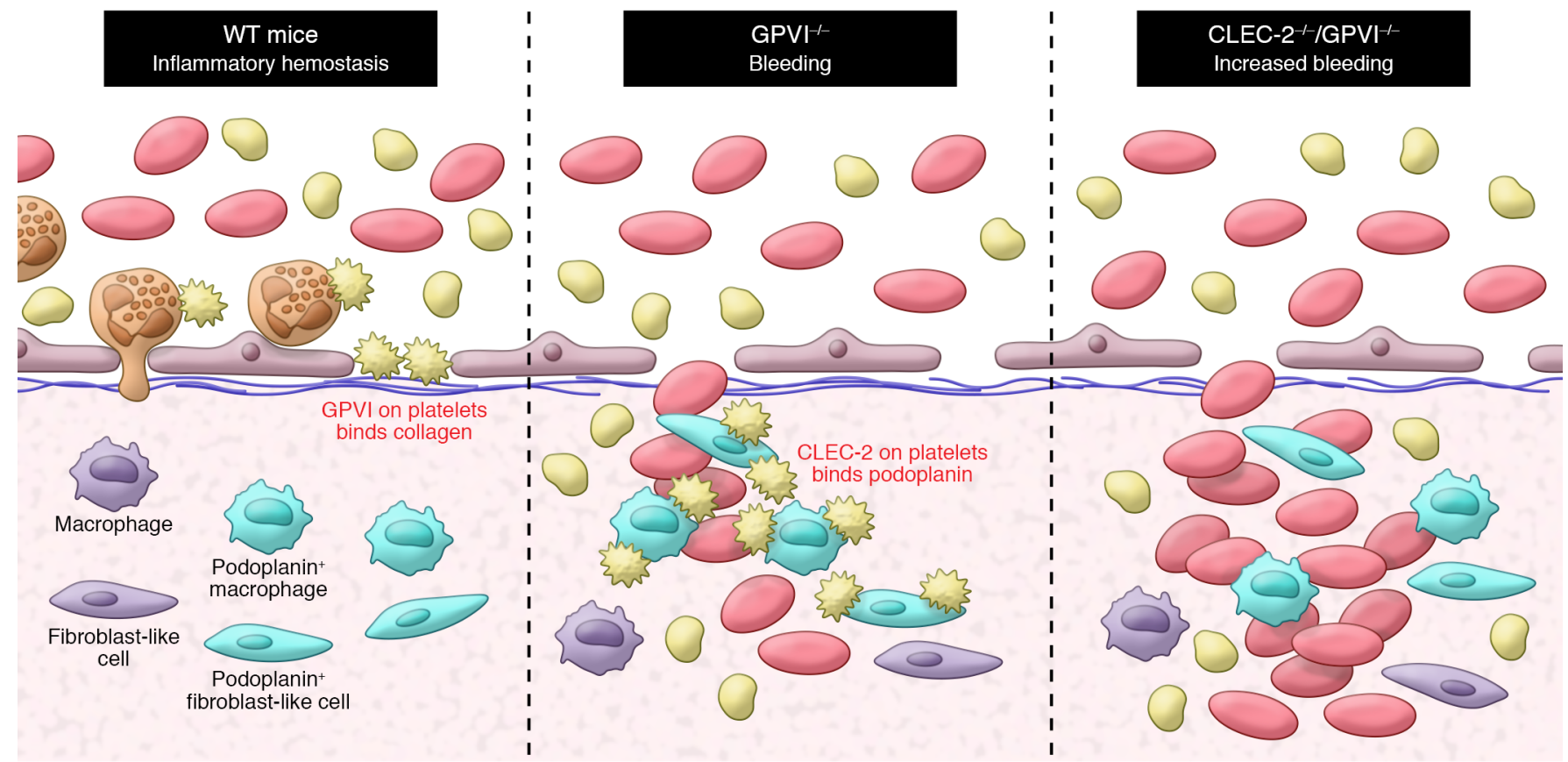

Figure 2. CLEC-2 and GPVI contribute to $\alpha_{\mathrm{II}} \boldsymbol{\beta}_{3}$-dependent and -independent hemostasis. (A) At the site of arterial injury, platelets roll, adhere, and aggregate to arrest bleeding through integrin $\alpha_{111} \beta_{3}$. The activation of GPVI by collagen drives integrin activation. Activated platelets release secondary mediators such as ADP and TxA, leading to recruitment of circulating platelets and formation of a platelet-fibrin-fibrinogen plug. The resulting thrombus is composed of a densely packed, fibrin-rich core and a looser outer shell of platelets. The diffusion of ligands from the core to the outer shell determines the size of the thrombus. Fibrin and fibrinogen bind to and activate GPVI, which may serve to propagate and stabilize the thrombus. (B) At the site of inflammation, platelets prevent hemorrhaging independent of $\alpha_{111} \beta_{3}$. The contribution of platelet receptors to the inflammatory hemostasis is stimulusand organ-dependent. During immune complex-mediated dermatitis, endothelial and stromal cell activation promotes recruitment, activation, and transmigration of neutrophils to the inflamed skin. Single platelets rapidly adhere and seal the neutrophil-mediated vascular damage through GPVI. The bleeding in GPVI-deficient mice is limited by the interaction of CLEC-2 with podoplanin on macrophages and stromal cells. In the absence of both receptors, a severe loss of vascular integrity is observed, with increased bleeding in the inflamed skin.

measurement of tail bleeding times in CLEC-2-deficient mice, although the limitations of these two assays should be acknowledged $(46,57,60,63)$. CLEC- 2 is not phosphorylated on a collagen surface under shear, consistent with its role as an adhesion receptor in the vasculature $(46,64,70)$. Mice deficient in the tyrosine kinase Syk or treated with the Syk inhibitor BI1002494 exhibit a negligible increase in tail bleeding times (71), whereas mice deficient in GPVI and CLEC-2 show a marked hemostatic defect (57) with the difference reflecting the adhesive role of CLEC-2.

These observations suggest that GPVI and CLEC-2 support hemostasis by functioning as signaling and adhesion receptors, respectively, at least in mice. 
Integrin $\alpha_{\mathrm{II}} \beta_{3}$-independent hemostasis. In a seminal study in thrombocytopenic mice, platelets were shown to play a role in prevention of bleeding in the skin, lungs, and brain during inflammatory challenge due to loss of vascular integrity (72). This inflammatory hemostasis is independent of most of the major platelet receptors, including integrin $\alpha_{\mathrm{II}} \beta_{3}$. The adoptive transfer of receptor-deficient platelets into thrombocytopenic IL4-RGPIb $\alpha$-transgenic mice identified GPVI and CLEC-2 as critical mediators of inflammatory hemostasis in the inflamed skin and lung (73). In contrast, in mice with a normal platelet count, inflammatory bleeding in the inflamed skin is only seen in the absence of GPVI, with mice deficient in both GPVI and CLEC-2 exhibiting increased bleeding (Figure 2B) $(74,75)$. The prevention of inflammatory bleeding in the skin is due to platelets sealing sites of breach in the vessel wall caused by neutrophils $(72,74,75)$. In contrast to the skin, bleeding in the inflamed lung in mice with a normal platelet count is independent of GPVI or CLEC-2, possibly due to redundancy with other receptors. Taken together, these results show that the contribution of platelet receptors to inflammatory hemostasis is organ and stimulus dependent (75). For an excellent review and further discussion, see ref. 76.

Another example of $\alpha_{\mathrm{IIb}} \beta_{3}$-independent hemostasis is seen in high endothelial venules in lymph nodes. In these vessels, immune cell trafficking (which increases during an active immune response) leads to bleeding in the presence of Src and Syk inhibitors or following post-developmental loss of podoplanin on fibroblastic reticular cells (FRCs) or CLEC-2 on platelets $(77,78)$. It has been proposed that podoplanin on the FRCs stimulates the release of sphingosine-1phosphate (S1P) from platelets, leading to increased VE-cadherin in endothelial cells (77), although the role of S1P has been questioned (79). Mice with a platelet-specific deletion of CLEC-2 are able to sustain a primary immune response but show a defect in immune cell recirculation after repeated immunizations (78).

The interaction of podoplanin and platelet CLEC-2 has been proposed to prevent retrograde flow of blood from the venous system by supporting activation of platelets on the lymphatic valves (80, 81). This would suggest that the lymphatic valves on their own are unable to form a sufficiently tight seal to counteract the higher pressure in the venous system. Retrograde blood flow is absent in mice or humans deficient in either of the integrin $\alpha_{\mathrm{IIb}} \beta_{3}$ subunits (82), making this a further example of $\alpha_{\mathrm{IIb}} \beta_{3}$-independent hemostasis.

The activation of CLEC- 2 by podoplanin also mediates hemostasis during development of the cerebrovasculature through a pathway that is largely independent of $\alpha_{\mathrm{IIb}} \beta_{3}$ (83). Mice deficient in podoplanin or platelet CLEC-2 have marked hemorrhaging in the mid- and hindbrain in mid-gestation, whereas only mild bleeding is seen in $\alpha_{\mathrm{IIb}} \beta_{3}$-deficient mice. Podoplanin is markedly upregulated on neuroepithelial cells at this stage of development, providing a mechanism of platelet activation. Mouse podoplanin binds to mouse CLEC-2 with low nanomolar affinity, allowing it to capture and retain platelets on immobilized podoplanin at arteriolar shear forces (84).

The above examples illustrate that $\alpha_{\mathrm{IIb}} \beta_{3}$-independent hemostasis is more widespread than previously recognized, representing a paradigm shift in our understanding of hemostatic mechanisms.

Arterial thrombosis. There is compelling evidence that GPVI is a significant contributor (along with other pathways) to arterial thrombosis following $\mathrm{FeCl}_{3}$-induced, laser, or mechanical injury, and upon atherosclerotic plaque rupture in mice $(85,86)$. Platelet activation by plaque material in vitro is also blocked by GPVI antibodies, dimeric GPVI (commercially available as Revacept), and inhibitors of platelet tyrosine kinases (87-91). The discovery of multiple ligands for GPVI, however, has brought uncertainty as to the mechanisms that drive thrombus formation. This is relevant to current phase II clinical trials with Revacept in patients with stable coronary artery disease or transient ischemic attacks (92). Revacept blocks platelet activation by collagen but not by fibrin $(36,40)$.

There are fewer studies on the role of CLEC- 2 in arterial thrombosis. Antibody depletion or genetic deletion of CLEC-2 from platelets reduces thrombus formation following $\mathrm{FeCl}_{3}$ induced, laser, or mechanical injury, with a greater effect in mice deficient in GPVI and CLEC-2 relative to loss of either receptor alone $(57,60,63)$. Thrombus formation induced by $\mathrm{FeCl}_{3}$ or photochemical injury is also inhibited in the presence of the Syk inhibitor BI1002494 or PRT-060318, or in Syk-deficient mice, with the degree of inhibition governed by the experimental model $(71,93)$.

These results highlight GPVI as a novel target for prevention of arterial thrombosis, while the potential of CLEC-2 as a target remains uncertain.

Thrombo-inflammation. Thrombo-inflammation was first used to describe the role of platelets in the inflammatory events that give rise to ischemia-reperfusion injury in a middle cerebral artery occlusion (MCAO) model of stroke (94). It is now used to describe the interplay between inflammatory and thrombotic pathomechanisms in arterial and venous disorders, including deep vein thrombosis (DVT) and in infection. In some cases, platelets drive the inflammatory events, and in others, the inflammation drives the thrombosis, with thrombo-inflammation being used to describe both sets of mechanisms. The term should not be confused with immunothrombosis, however, which refers to capture of pathogens in the vasculature to facilitate their removal and limit dissemination (95).

GPVI is a key player in the neuronal damage that occurs following cerebral reperfusion in the MCAO model of stroke (96), as shown by the beneficial effect of GPVI antibodies or Revacept (9799). The reperfusion triggers an inflammatory response involving platelet adhesion/activation mechanisms, T cells, neutrophils, and monocytes, leading to tissue damage and late-stage thrombus formation. Inhibiting thrombus formation with $\alpha_{\mathrm{IIb}} \beta_{3}$ blockers dramatically increases intracranial hemorrhage and infarct growth (96), suggesting that the beneficial effect of GPVI blockade is due to reduction of the inflammatory response, possibly by reducing release of IL-1 $\beta$ and polyphosphates and attenuating inflammatory cell recruitment $(94,100)$. Similarly, GPVI blockade causes a reduction in inflammatory cell recruitment and infarct size following myocardial ischemia-reperfusion injury (101). Inhibition of GPVI also increases perfusion, although this is accompanied by increased intramyocardial bleeding. Separating the role of GPVI in inflammation and thrombotic events is required to fully understand its role in thrombo-inflammation.

The podoplanin/CLEC-2 axis is thought to be a major player in thrombo-inflammatory disorders as a result of upregulation of podoplanin on stromal cells and tissue-resident macrophages. A modified strain of Salmonella typhimurium leads to a delayed 
WT
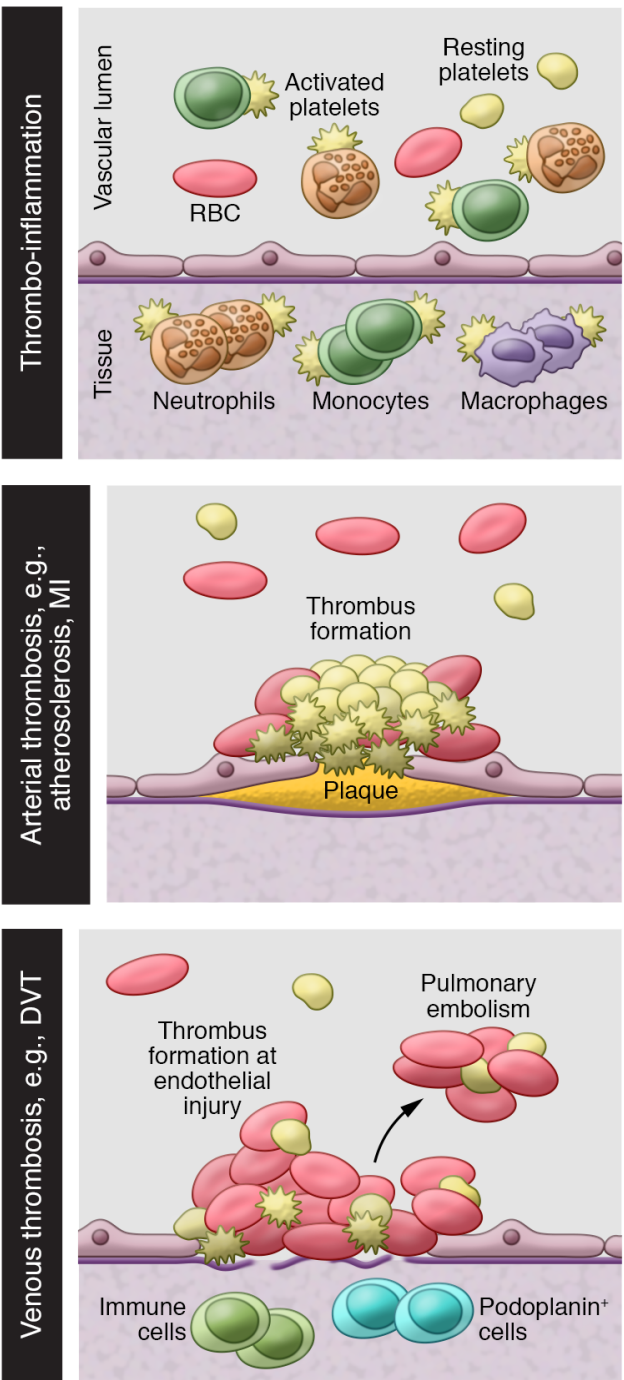

Gp6
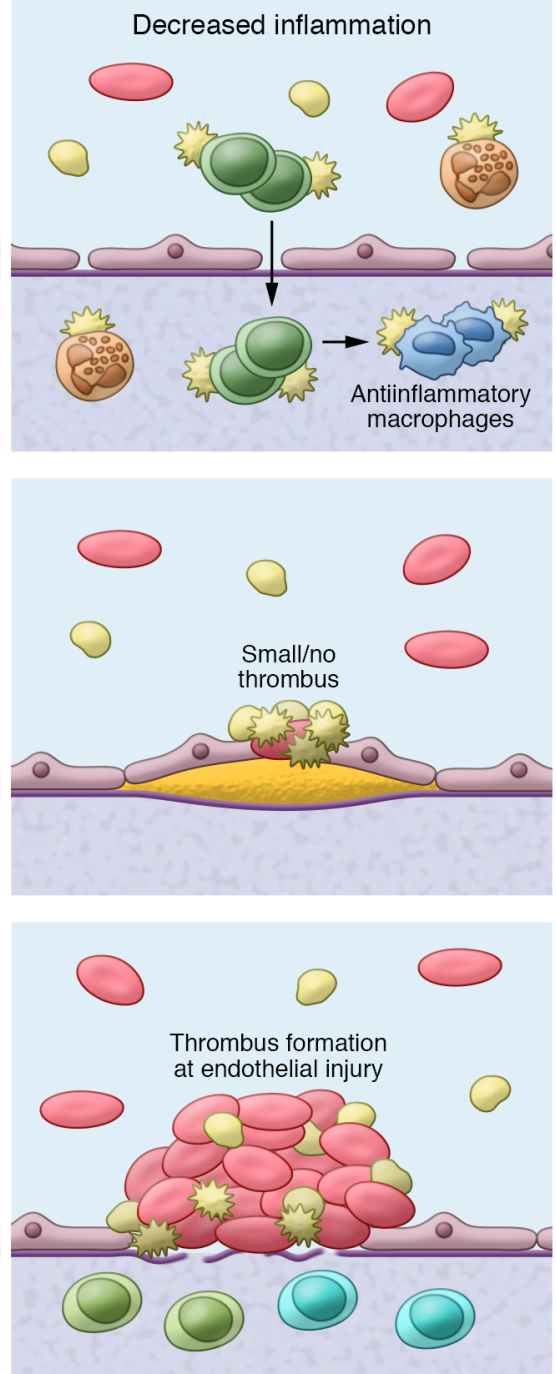

Clectb
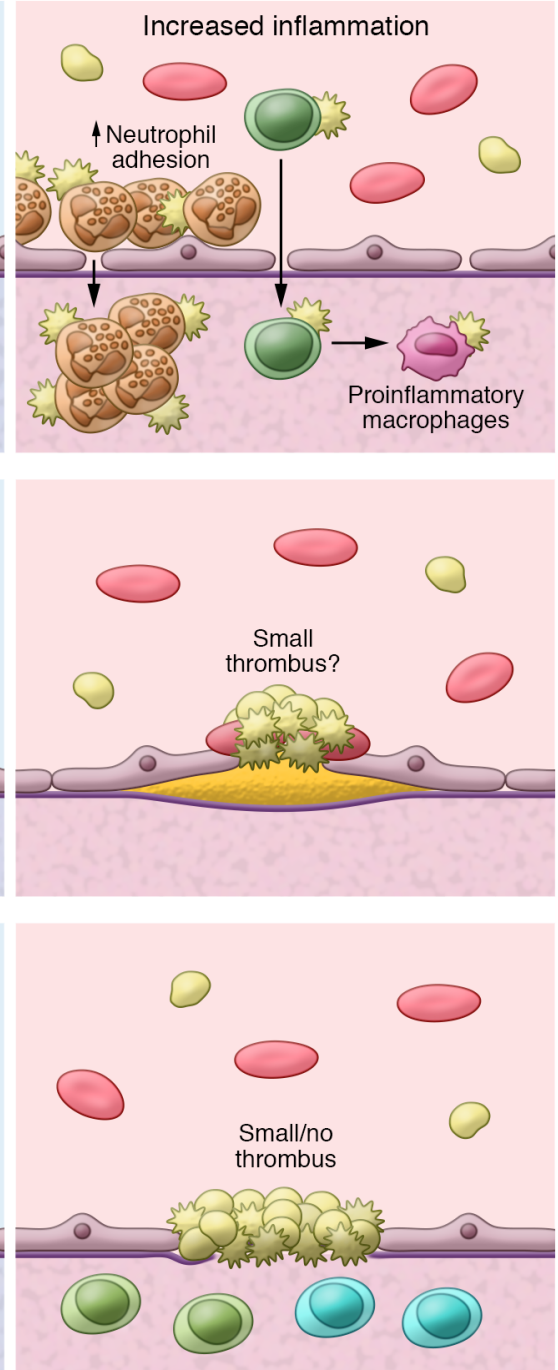

Figure 3. Multiple roles of CLEC-2 and GPVI in thrombo-inflammation and thrombosis. During thrombo-inflammatory diseases, platelets regulate immune cell recruitment at the site of inflammation. Deletion of GPVI $\left(\mathrm{Cp}^{-{ }^{--}}\right)$decreases the inflammatory reaction as a consequence of a reduction in neutrophil recruitment and the number of inflammatory macrophages. Conversely, deletion of CLEC2 (Clec1b $\left.{ }^{-1}\right)$ potentiates the inflammatory reaction through an increase in neutrophil infiltration and inducing a proinflammatory macrophage phenotype. In addition, GPVI promotes the underlying inflammation that drives plaque formation and drives thrombus formation upon plaque rupture. During venous thrombosis, CLEC- 2 promotes thrombosis at sites of breach in the vessel wall by upregulating podoplanin on stromal cells and inflammatory macrophages.

platelet/fibrin-rich thrombosis in the liver at the time that the bacterial load is in decline (102). Upregulation of podoplanin in inflammatory foci and subendothelial layers as well as intravascular thrombosis are abolished in mice deficient in TLR4 and IFN- $\gamma$, showing that it is the inflammatory response that gives rise to the thrombosis. Thrombosis is inhibited in CLEC-2-deficient mice, suggesting that exposure of podoplanin at sites of breach in the vessel wall drives CLEC-2-dependent platelet activation.

DVT is also caused by inflammatory events in the vessel wall, leading to a thrombus that is rich in red cells and fibrin $(103,104)$. Thrombus formation involves the interaction of von Willebrand factor (vWF), platelets, neutrophils, and mast cells, and upregulation of podoplanin in the vessel wall (104-106). Mice deficient in CLEC-2 or treated with an anti-podoplanin antibody show a reduction in thrombus formation (107).
The recognition of the interplay between inflammation and platelets opens up new opportunities for therapeutic intervention at either the level of GPVI and CLEC-2 or the inflammatory events.

\section{Sterile and non-sterile injury}

The regulation of the inflammatory response is crucial in limiting tissue damage and promoting repair following injury. In inflammation's initial phase, platelet activation promotes recruitment and activation of neutrophils and monocytes, potentiating the inflammatory response. (108). In later stages, platelets may help in the resolution of inflammation through inhibition of proinflammatory macrophages and monocytes $(109,110)$. GPVI and CLEC-2 are emerging as key mediators of both phases via the regulation of leukocyte activation and recruitment (Figure 3). In particular, the upregulation of podoplanin at sites of infection fuels speculation that CLEC- 2 is involved, 
although it should be remembered that podoplanin signals constitutively and has additional ligands $(50,111)$.

Bacterial infection. Platelets play a critical role in the inflammatory response associated with infection, as illustrated by the increase in major organ damage seen in thrombocytopenic mice following bacterial infection or lipopolysaccharide-induced inflammation $(109,112)$. As yet, however, there are only a handful of studies on the platelet receptors involved.

GPVI but not CLEC-2 has been reported to be protective in a mouse model of Klebsiella pneumonia-mediated pneumosepsis (113). GPVI deficiency increases bacterial load in the lungs and distant organs in association with a reduction in circulating platelets and platelet-leukocyte aggregates. The activation of GPVI promotes formation of platelet-leukocyte aggregates, leading to enhanced phagocytosis of Klebsiella pneumonia in vitro (113), although it is not known whether this occurs in vivo. GPVI has been shown to mediate platelet activation by Helicobacter pylori and Staphylococcus aureus $(114,115)$, but there are no reports of activation by Klebsiella pneumonia. Conversely, the absence of CLEC-2 but not GPVI increases clinical severity in a cecal ligation and puncture (CLP) model or following injection of lipopolysaccharide (116). Deletion of CLEC-2 exacerbates the cytokine storm and inhibits inflammatory macrophage recruitment to the infected peritoneum, resulting in increased bacterial load and organ injury (116). The absence of CLEC-2 also accelerates the decline in lung function in a lipopolysaccharide-induced acute respiratory distress syndrome (ARDS) model due to an increase in neutrophil recruitment to the inflamed lung (59). Crosslinking podoplanin using mAb 8.1.1 is protective against lung injury but not in systemic endotoxemia or CLP $(116,117)$, suggesting that the beneficial effect of targeting podoplanin depends on the stimulus, organ, and role of other immune cells. Therefore, although they share a common signaling pathway, the selective contribution of CLEC-2 and GPVI to inflammation and infection is tightly regulated by their ligands and the underlying mechanisms.

Viral infection. More than ten years ago, GPVI and CLEC-2 were reported to bind directly to and promote transport and dissemination of hepatitis C virus (118) and HIV-1 (119), respectively, but the clinical significance remains uncertain.

Sterile injury. Attention has been paid to the role of GPVI in sterile inflammation due to the seminal study showing that GPVI promotes accumulation of proinflammatory plateletderived microvesicles in rheumatoid arthritis, with GPVI-deficient mice exhibiting reduced clinical signs and tissue pathology $(120,121)$. The identity of the GPVI-activating ligand and the mechanism underlying the entry of microvesicles into the joints are unknown. GPVI has also been shown to promote platelet recruitment to inflamed glomeruli during immune complexmediated glomerulonephritis, potentiating neutrophil recruitment (122). In dermatitis, GPVI was shown to promote the proinflammatory phenotype of macrophages, with loss of GPVI associated with a switch toward a pro-resolution M2 phenotype (123). In arthritis, increased podoplanin expression in areas of hyperplasia, inflammation, and disrupted tissue structure is indicative of a role in the inflammatory process (124-126), with podoplanin upregulation occurring on several cell types, including fibroblasts, macrophages, and Th17 T cells (124-128).
Podoplanin and CLEC-2 have been reported to be antiinflammatory in an experimental autoimmune encephalomyelitis (EAE) model in mice. An increase in EAE severity is seen on deletion of podoplanin or platelet CLEC-2, and EAE is reduced upon overexpression of podoplanin on Th17 cells associated with an increased rate of resolution of inflammation, suggesting that podoplanin is a nonpathogenic marker of Th17 cells and that the podoplanin/CLEC-2 axis promotes resolution (129-131). In contrast, overexpression of podoplanin on Th17 T cells and fibroblasts correlates with increased inflammation and clinical severity in rheumatoid arthritis (132), showing that the beneficial role of podoplanin is disease dependent.

CLEC-2 has also been shown to have a beneficial role in a diabetic mouse model through an interaction with podoplaninpositive Kupffer cells. The injection of recombinant dimeric CLEC-2 promoted an antiinflammatory phenotype, altering expression of genes involved in lipid metabolism, reducing blood glucose and liver triglyceride levels, and improving glucose tolerance (133). The mechanism underlying the beneficial effect of CLEC-2, however, is unclear.

These studies reveal both pro- and antiinflammatory roles of GPVI and CLEC-2, respectively, in sterile injury. Exploiting this in the clinic will require a greater understanding of the mechanisms of activation and function of the two ITAM receptors.

Tissue repair. The presence of podoplanin on epidermal keratinocytes suggests a role in tissue repair. In an in vitro model, siRNAmediated downregulation of podoplanin in epidermal keratinocytes decreased migration in association with upregulation of E-cadherin (134). Treatment with platelets or addition of recombinant CLEC-2 inhibits keratinocyte migration and increases E-cadherin expression (134). These results need to be confirmed in vivo.

\section{Cancer}

Platelets play a key role in tumor growth and metastasis by supporting blood and lymphatic vessel formation and aiding cancer cells in evading the immune system and metastasizing $(135,136)$. Moreover, tumoral activation of platelets can lead to pathological thrombosis and thromboembolism that further complicate treatment (137). Relatively little is known about the role of GPVI in cancer, although it has been shown to promote metastasis in lung cancer and melanoma models (138). In contrast, the role of podoplanin in cancer has been studied for more than 15 years, and several strategies to inhibit podoplanin have been developed.

Podoplanin is expressed at the invasive front of many tumors and on cancer-associated fibroblasts, with high levels of expression associated with poor outcome in glioma, squamous cell carcinoma, mesothelioma, and melanoma (139-141). Cytokines induce podoplanin expression, which in some cases leads to further cytokine production as well as metalloproteinase expression, facilitating tumor cell invasion. In mouse models, podoplanin promotes tumor growth, epithelial-mesenchymal transition (EMT), invasion, and metastasis $(50,139,142)$. Many of these actions are mediated through platelet CLEC-2, including tumor progression, metastasis, and cancer-induced thrombosis (143-146). The podoplanin/CLEC-2 axis promotes an immunosuppressed microenvironment, facilitating spread and growth of the cancer (131). Blocking podoplanin with NZ1 (anti-human) or 8.1.1 (anti-mouse) mAb, 
or podoplanin-targeting CAR T cells (NZ-1-CAR T cells), has been shown to inhibit cancer progression (147-149). A similar beneficial effect has been reported using a small molecule inhibitor of CLEC-2, 2CP (150), or mutated rhodocytin in an experimental lung metastasis model (147). Moreover, aberrant glycosylation of podoplanin in cancer cells raises the possibility of a selective therapeutic targeting strategy (151).

In contrast, the expression of CLEC-2 on cancer cells in hepatocellular carcinoma and gastric cancer is protective. Downregulation of CLEC-2 has been observed on the invasive form of both cancers, and has been linked to increased EMT and tumor aggressiveness $(152,153)$. Therefore, the role of CLEC-2 of in cancer progression and metastasis depends on its expression on cancer cells or platelets.

\section{Development}

There is no evidence to suggest that GPVI plays a role in development, although it is noteworthy that neonatal platelets are hyporesponsive to GPVI and CLEC-2 for reasons that are not yet known (154). In contrast, the podoplanin/CLEC-2 axis plays a critical role in several stages of embryonic development. Mice with a constitutive deficiency in CLEC- 2 or podoplanin have blood-lymphatic mixing and die at the time of birth as described above $(58,80,82,155)$. A similar phenotype is seen in a CLEC-2 signaling-null mouse (64) and in mice deficient in Syk, SLP-76, and PLC $\gamma 2$, demonstrating that this is caused by loss of platelet activation by CLEC-2 (156-159). Blood-lymphatic mixing is also present in mouse embryos with other defects in platelet signaling pathways or severe thrombocytopenia $(58,80,82,155,160$, 161). The mechanism of the blood-lymphatic mixing, however, is uncertain, although it is not due to a failure of Prox-1-positive endothelial cells to migrate away from the cardinal vein, as initially proposed (162). The binding of podoplanin to CLEC-2 leads to clustering of both receptors, resulting in stable adhesion of platelets to lymphatic endothelial cells and inhibition of podoplanin signaling $(58,163)$. This may prevent fusion of migrating lymphatic endothelial cells with blood vessels (155).

Additional developmental defects are present in constitutive CLEC-2- and podoplanin-deficient mice, including defects in the development of the cerebrovasculature (discussed above), the absence of mesenteric and inguinal lymph nodes, and a reduction in platelet count $(58,78,155,164,165)$. The presence of lymph nodes in the PF4-Cre.Clec- $1 b^{f l / f l}$ mouse demonstrates, however, that platelet CLEC-2 is dispensable for their formation. Mice with post-developmental deletion of platelet CLEC-2 have a normal platelet count, suggesting that the mild thrombocytopenia occurs secondary to blood-lymphatic mixing. The absence of a phenotype upon deletion of podoplanin on lung type 1 alveolar cells and kidney podocytes demonstrates that the podoplanin/CLEC-2 axis is not required for development of these two organs $(59,116,166,167)$.

\section{Concluding remarks}

Most of our knowledge on GPVI and CLEC-2 in disease is based on in vitro data and experimental models in mice and not humans. Nevertheless, the relatively minor or negligible roles of GPVI and CLEC-2 in hemostasis and their contribution to thrombosis and thrombo-inflammation identify both receptors as targets for development of a new class of antithrombotic drugs. Moreover, the presence of two haplotypes for GPVI - GPVIa and GPVIb and the association between GPVI haplotypes and pathological conditions such as stroke and myocardial infarction strengthen the potential of anti-GPVI therapy (168-170). While bleeding is a concern with GPVI-targeting strategies, CLEC-2 does not appear to play a major role in hemostasis. Inflammatory bleeding and embolization are also a concern in targeting GPVI, although the absence of reports of bleeding at sites of inflammation in GPVI-deficient patients suggests that this may be overstated. The risk of bleeding is increased in the presence of other antiplatelet agents such as aspirin (68), and clinical trials will have to take this into account in targeting GPVI or CLEC-2. The interaction of CLEC-2 and podoplanin is also a target in treatment of non-hemostatic disorders, including in chronic inflammatory conditions and infection, and in reducing cancer metastasis. However, targeting GPVI and CLEC-2 in disease will have to be closely monitored due to their multiple roles in regulating platelet function during disease progression, while a greater understanding of the role of the various ligands for GPVI and CLEC-2 may pave the way for development of receptor-selective ligands. Humanized GPVI and CLEC-2 mice represent a powerful tool to evaluate the effect of anti-GPVI and anti-CLEC-2 agents in different thrombotic and inflammatory diseases (171).

The challenge is now to translate findings from mouse to human, with encouraging results from the phase II trials with Revacept and the human GPVI-blocking F(ab) ACT-017 anticipated to drive further interest in this field. Inhibitors of Src, Syk, and Tec tyrosine kinases are already in the clinic and could be used to block platelet activation by GPVI and CLEC-2. While there is currently concern about the effect of these inhibitors on bleeding, this may be overestimated due to off-target actions (172). Further, the efficacy of the irreversible Btk inhibitor ibrutinib, which like aspirin can be used in a low-dose pharmaceutical preparation, against platelet activation by human plaque material offers considerable hope in this regard (91). The next few years will see a deeper understanding of the roles of GPVI and CLEC-2 in this remarkable cell and possible translation to the clinic.

\section{Acknowledgments}

SPW acknowledges support from the British Heart Foundation (BHF), Wellcome Trust, and Medical Research Council (MRC). JR is supported by the BHF (RG/13/18/30563). BN is supported by the Deutsche Forschungsgemeinschaft (SFB/TR240) and the Rudolf Virchow Center. We thank Alexander Brill and Elizabeth Haining for critical appraisal of the manuscript.

Address correspondence to: Bernhard Nieswandt, Institute of Experimental Biomedicine, University Hospital and Rudolf Virchow Centre, University of Würzburg, Josef-Schneider-Str 2, 97080 Würzburg, Germany. Phone: 49.931.31.80405; Email: bernhard.nieswandt@virchow.uni-wuerzburg.de. Or to: Steve P. Watson, Institute of Cardiovascular Sciences, IBR Building, College of Medical and Dental Sciences, University of Birmingham, Edgbaston, Birmingham B15 2TT, United Kingdom. Phone: 44.121.4146514, Email: s.p.watson@bham.ac.uk. 
1. Nieswandt B, Watson SP. Platelet-collagen interaction: is GPVI the central receptor? Blood. 2003;102(2):449-461.

2. Moroi M, Jung SM. Platelet glycoprotein VI: its structure and function. Thromb Res. 2004;114(4):221-233.

3. Best D, et al. GPVI levels in platelets: relationship to platelet function at high shear. Blood. 2003;102(8):2811-2818.

4. Ezumi Y, Uchiyama T, Takayama H. Molecular cloning, genomic structure, chromosomal localization, and alternative splice forms of the platelet collagen receptor glycoprotein VI. Biochem Biophys Res Commun. 2000;277(1):27-36.

5. Miura Y, Takahashi T, Jung SM, Moroi M. Analysis of the interaction of platelet collagen receptor glycoprotein VI (GPVI) with collagen. A dimeric form of GPVI, but not the monomeric form, shows affinity to fibrous collagen. J Biol Chem. 2002;277(48):46197-46204.

6. Herr AB, Farndale RW. Structural insights into the interactions between platelet receptors and fibrillar collagen. J Biol Chem. 2009;284(30):19781-19785.

7. Horii K, Kahn ML, Herr AB. Structural basis for platelet collagen responses by the immune-type receptor glycoprotein VI. Blood. 2006;108(3):936-942

8. Jung SM, et al. Constitutive dimerization of glycoprotein VI (GPVI) in resting platelets is essential for binding to collagen and activation in flowing blood. J Biol Chem. 2012;287(35):30000-30013.

9. Loyau S, et al. Platelet glycoprotein VI dimerization, an active process inducing receptor competence, is an indicator of platelet reactivity. Arterioscler Thromb Vasc Biol. 2012;32(3):778-785.

10. Poulter NS, et al. Clustering of glycoprotein VI (GPVI) dimers upon adhesion to collagen as a mechanism to regulate GPVI signaling in platelets. J Thromb Haemost. 2017;15(3):549-564.

11. Arthur JF, Dunkley S, Andrews RK. Platelet glycoprotein VI-related clinical defects. Br J Haematol. 2007;139(3):363-372.

12. Matus V, et al. An adenine insertion in exon 6 of human GP6 generates a truncated protein associated with a bleeding disorder in four Chilean families. J Thromb Haemost. 2013;11(9):1751-1759.

13. Kato K, et al. The contribution of glycoprotein VI to stable platelet adhesion and thrombus formation illustrated by targeted gene deletion. Blood. 2003;102(5):1701-1707.

14. Nieswandt B, Bergmeier W, Schulte V, Rackebrandt K, Gessner JE, Zirngibl H. Expression and function of the mouse collagen receptor glycoprotein VI is strictly dependent on its association with the FcRgamma chain.J Biol Chem. 2000;275(31):23998-24002.

15. Nieswandt B, et al. Long-term antithrombotic protection by in vivo depletion of platelet glycoprotein VI in mice. JExp Med. 2001;193(4):459-469.

16. Stegner D, Popp M, Lorenz V, Wax JK, Gessner JE, Nieswandt B. Fc $\gamma$ RIIB on liver sinusoidal endothelial cells is essential for antibodyinduced GPVI ectodomain shedding in mice. Blood. 2016;128(6):862-865.

17. Boylan B, et al. Anti-GPVI-associated ITP: an acquired platelet disorder caused by autoantibody-mediated clearance of the GPVI/FcR- gamma-chain complex from the human platelet surface. Blood. 2004;104(5):1350-1355.

18. Gardiner EE, Karunakaran D, Shen Y, Arthur JF, Andrews RK, Berndt MC. Controlled shedding of platelet glycoprotein (GP)VI and GPIb-IX-V by ADAM family metalloproteinases. J Thromb Haemost. 2007;5(7):1530-1537.

19. Montague SJ, et al. Soluble GPVI is elevated in injured patients: shedding is mediated by fibrin activation of GPVI. Blood Adv. 2018;2(3):240-251.

20. Coxon CH, Geer MJ, Senis YA. ITIM receptors: more than just inhibitors of platelet activation. Blood. 2017;129(26):3407-3418.

21. Mazharian A, et al. Mice lacking the ITIMcontaining receptor G6b-B exhibit macrothrombocytopenia and aberrant platelet function. $\mathrm{Sci}$ Signal. 2012;5(248):ra78.

22. Hofmann I, et al. Congenital macrothrombocytopenia with focal myelofibrosis due to mutations in human G6b-B is rescued in humanized mice. Blood. 2018;132(13):1399-1412.

23. Gitz E, et al. CLEC-2 expression is maintained on activated platelets and on platelet microparticles. Blood. 2014;124(14):2262-2270.

24. Al-Tamimi M, et al. Soluble glycoprotein VI is raised in the plasma of patients with acute ischemic stroke. Stroke. 2011;42(2):498-500.

25. Stack JR, et al. Soluble glycoprotein VI, a specific marker of platelet activation is increased in the plasma of subjects with seropositive rheumatoid arthritis. PLOS ONE. 2017;12(11):e0188027.

26. Egan K, et al. Increased soluble GPVI levels in cirrhosis: evidence for early in vivo platelet activation. J Thromb Thrombolysis. 2017;43(1):54-59.

27. Inoue $\mathrm{O}$, et al. Laminin stimulates spreading of platelets through integrin alpha6beta1dependent activation of GPVI. Blood. 2006;107(4):1405-1412

28. Schaff $\mathrm{M}$, et al. Integrin $\alpha 6 \beta 1$ is the main receptor for vascular laminins and plays a role in platelet adhesion, activation, and arterial thrombosis. Circulation. 2013;128(5):541-552.

29. Bültmann A, et al. Impact of glycoprotein VI and platelet adhesion on atherosclerosis - a possible role of fibronectin. J Mol Cell Cardiol. 2010;49(3):532-542.

30. Schönberger T, et al. The dimeric platelet collagen receptor GPVI-Fc reduces platelet adhesion to activated endothelium and preserves myocardial function after transient ischemia in mice. $\mathrm{Am}$ J Physiol, Cell Physiol. 2012;303(7):C757-C766.

31. Schulz C, et al. EMMPRIN (CD147/basigin) mediates platelet-monocyte interactions in vivo and augments monocyte recruitment to the vascular wall. J Thromb Haemost. 2011;9(5):1007-1019.

32. Riba R, Hughes CE, Graham A, Watson SP, Naseem KM. Globular adiponectin induces platelet activation through the collagen receptor GPVI-Fc receptor gamma chain complex. J Thromb Haemost. 2008;6(6):1012-1020.

33. Elaskalani O, et al. Oligomeric and fibrillar amyloid beta 42 induce platelet aggregation partially through GPVI. Platelets. 2018;29(4):415-420.

34. Alshehri OM, et al. Fibrin activates GPVI in human and mouse platelets. Blood. 2015;126(13):1601-1608.

35. Mammadova-Bach E, et al. Platelet glycoprotein VI binds to polymerized fibrin and promotes thrombin generation. Blood. 2015;126(5):683-691.

36. Onselaer MB, et al. Fibrin and D-dimer bind to monomeric GPVI. Blood Adv. 2017;1(19):1495-1504.

37. Mangin PH, et al. Immobilized fibrinogen activates human platelets through glycoprotein VI. Haematologica. 2018;103(5):898-907.

38. Ebrahim M, et al. Dimeric glycoprotein VI binds to collagen but not to fibrin. Thromb Haemost. 2018;118(2):351-361.

39. Slater A, et al. Does fibrin(ogen) bind to monomeric or dimeric GPVI, or not at all? [published online ahead of print August 15, 2018]. Platelets. https:// doi.org/10.1080/09537104.2018.1508649.

40. Induruwa I, et al. Platelet collagen receptor glycoprotein VI-dimer recognizes fibrinogen and fibrin through their D-domains, contributing to platelet adhesion and activation during thrombus formation. J Thromb Haemost. 2018;16(2):389-404.

41. Lehmann M, et al. Platelets drive thrombus propagation in a hematocrit and glycoprotein VI-dependent manner in an in vitro venous thrombosis model. Arterioscler Thromb Vasc Biol. 2018;38(5):1052-1062.

42. Alshehri OM, et al. Activation of glycoprotein VI (GPVI) and C-type lectin-like receptor-2 (CLEC-2) underlies platelet activation by diesel exhaust particles and other charged/hydrophobic ligands. Biochem J. 2015;468(3):459-473.

43. Flierl U, et al. Phosphorothioate backbone modifications of nucleotide-based drugs are potent platelet activators. J Exp Med. 2015;212(2):129-137.

44. Watson AA, Brown J, Harlos K, Eble JA, Walter TS, O'Callaghan CA. The crystal structure and mutational binding analysis of the extracellular domain of the platelet-activating receptor CLEC-2. J Biol Chem . 2007;282(5):3165-3172.

45. Watson SP, Herbert JM, Pollitt AY. GPVI and CLEC-2 in hemostasis and vascular integrity. J Thromb Haemost. 2010;8(7):1456-1467.

46. Hughes CE, Navarro-Núñez L, Finney BA, Mourão-Sá D, Pollitt AY, Watson SP. CLEC-2 is not required for platelet aggregation at arteriolar shear. J Thromb Haemost. 2010;8(10):2328-2332.

47. Lowe KL, et al. The expression of mouse CLEC-2 on leucocyte subsets varies according to their anatomical location and inflammatory state. Eur J Immunol. 2015;45(9):2484-2493.

48. Burkhart JM, et al. The first comprehensive and quantitative analysis of human platelet protein composition allows the comparative analysis of structural and functional pathways. Blood. 2012;120(15):e73-e82.

49. Zeiler M, Moser M, Mann M. Copy number analysis of the murine platelet proteome spanning the complete abundance range. Mol Cell Proteomics. 2014;13(12):3435-3445.

50. Astarita JL, Acton SE, Turley SJ. Podoplanin: emerging functions in development, the immune system, and cancer. Front Immunol. 2012;3:283.

51. Krishnan H, et al. Serines in the intracellular tail of podoplanin (PDPN) regulate cell motility. J Biol Chem. 2013;288(17):12215-12221.

52. Krishnan H, et al. PKA and CDK5 can phosphorylate specific serines on the intracellular domain of podoplanin (PDPN) to inhibit cell motility. Exp Cell Res. 2015;335(1):115-122.

53. Kato Y, et al. Molecular identification of Aggrus/ 
T1alpha as a platelet aggregation-inducing factor expressed in colorectal tumors. J Biol Chem. 2003;278(51):51599-51605.

54. Kaneko MK, et al. Functional glycosylation of human podoplanin: glycan structure of platelet aggregation-inducing factor. FEBS Lett. 2007;581(2):331-336.

55. Nagae M, Morita-Matsumoto K, Kato M, Kaneko MK, Kato Y, Yamaguchi Y. A platform of C-type lectin-like receptor CLEC-2 for binding O-glycosylated podoplanin and nonglycosylated rhodocytin. Structure. 2014;22(12):1711-1721.

56 . Tsukiji N, et al. Platelets play an essential role in murine lung development through Clec-2/podoplanin interaction. Blood. 2018;132(11):1167-1179.

57. Bender $\mathrm{M}$, et al. Combined in vivo depletion of glycoprotein VI and C-type lectin-like receptor 2 severely compromises hemostasis and abrogates arterial thrombosis in mice. Arterioscler Thromb Vasc Biol. 2013;33(5):926-934.

58. Finney BA, et al. CLEC-2 and Syk in the megakaryocytic/platelet lineage are essential for development. Blood. 2012;119(7):1747-1756.

59. Lax S, et al. Platelet CLEC-2 protects against lung injury via effects of its ligand podoplanin on inflammatory alveolar macrophages in the mouse. Am J Physiol Lung Cell Mol Physiol. 2017;313(6):L1016-L1029.

60. May F, et al. CLEC-2 is an essential plateletactivating receptor in hemostasis and thrombosis. Blood. 2009;114(16):3464-3472.

61. Lorenz V, et al. Targeted downregulation of platelet CLEC-2 occurs through Syk-independent internalization. Blood. 2015;125(26):4069-4077.

62. Kazama F, et al. Measurement of soluble C-type lectin-like receptor 2 in human plasma. Platelets. 2015;26(8):711-719.

63. Suzuki-Inoue $\mathrm{K}$, et al. Essential in vivo roles of the C-type lectin receptor CLEC-2: embryonic/ neonatal lethality of CLEC-2-deficient mice by blood/lymphatic misconnections and impaired thrombus formation of CLEC-2-deficient platelets. J Biol Chem. 2010;285(32):24494-24507.

64. Haining EJ, et al. CLEC-2 contributes to hemostasis independently of classical hemITAM signaling in mice. Blood. 2017;130(20):2224-2228.

65. Manne BK, et al. Fucoidan is a novel platelet agonist for the C-type lectin-like receptor 2 (CLEC-2) J Biol Chem. 2013;288(11):7717-7726.

66. Stalker TJ, et al. A systems approach to hemostasis: 3. thrombus consolidation regulates intrathrombus solute transport and local thrombin activity. Blood. 2014;124(11):1824-1831.

67. Bynagari-Settipalli YS, et al. Redundancy and interaction of thrombin- and collagen-mediated platelet activation in tail bleeding and carotid thrombosis in mice. Arterioscler Thromb Vasc Biol. 2014;34(12):2563-2569.

68. Gruner S, et al. Anti-glycoprotein VI treatment severely compromises hemostasis in mice with reduced alpha2beta1 levels or concomitant aspirin therapy. Circulation. 2004;110(18):2946-2951.

69. Dütting S, et al. Growth factor receptor-bound protein 2 contributes to (hem)immunoreceptor tyrosine-based activation motif-mediated signaling in platelets. Circ Res. 2014;114(3):444-453.

70. Hughes CE, et al. CLEC-2 activates Syk through dimerization. Blood. 2010;115(14):2947-2955
71. van Eeuwijk JM, et al. The novel oral Syk inhibitor, Bl1002494, protects mice from arterial thrombosis and thromboinflammatory brain infarction. Arterioscler Thromb Vasc Biol. 2016;36(6):1247-1253.

72. Goerge T, et al. Inflammation induces hemorrhage in thrombocytopenia. Blood. 2008;111(10):4958-4964

73. Boulaftali Y, et al. Platelet ITAM signaling is critical for vascular integrity in inflammation. J Clin Invest. 2013;123(2):908-916.

74. Gros A, et al. Single platelets seal neutrophilinduced vascular breaches via GPVI during immune-complex-mediated inflammation in mice. Blood. 2015;126(8):1017-1026.

75. Rayes J, et al. The contribution of platelet glycoprotein receptors to inflammatory bleeding prevention is stimulus and organ dependent. Haematologica. 2018;103(6):e256-e258.

76. Boulaftali Y, Mawhin MA, Jandrot-Perrus M, Ho-Tin-Noé B. Glycoprotein VI in securing vascular integrity in inflamed vessels. Res Pract Thromb Haemost. 2018;2(2):228-239.

77. Herzog BH, et al. Podoplanin maintains high endothelial venule integrity by interacting with platelet CLEC-2. Nature. 2013;502(7469):105-109.

78. Bénézech C, et al. CLEC-2 is required for development and maintenance of lymph nodes. Blood 2014;123(20):3200-3207.

79. Gazit SL, et al. Platelet and erythrocyte sources of S1P are redundant for vascular development and homeostasis, but both rendered essential after plasma S1P depletion in anaphylactic shock. Circ Res. 2016;119(8):e110-e126.

80. Hess PR, et al. Platelets mediate lymphovenous hemostasis to maintain blood-lymphatic separation throughout life. J Clin Invest. 2014;124(1):273-284.

81. Bianchi R, et al. Postnatal deletion of podoplanin in lymphatic endothelium results in blood filling of the lymphatic system and impairs dendritic cell migration to lymph nodes. Arterioscler Thromb Vasc Biol. 2017;37(1):108-117.

82. Uhrin $P$, et al. Novel function for blood platelets and podoplanin in developmental separation of blood and lymphatic circulation. Blood. 2010;115(19):3997-4005.

83. Lowe KL, et al. Podoplanin and CLEC-2 drive cerebrovascular patterning and integrity during development. Blood. 2015;125(24):3769-3777.

84. Lombard SE, et al. Mouse podoplanin supports adhesion and aggregation of platelets under arterial shear: a novel mechanism of haemostasis. Platelets. 2018;29(7):716-722.

85. Dütting S, Bender M, Nieswandt B. Platelet GPVI: a target for antithrombotic therapy?! Trends Pharmacol Sci. 2012;33(11):583-590.

86. Massberg S, et al. A crucial role of glycoprotein VI for platelet recruitment to the injured arterial wall in vivo. J Exp Med. 2003;197(1):41-49.

87. Penz S, et al. Human atheromatous plaques stimulate thrombus formation by activating platelet glycoprotein VI. FASEB J. 2005;19(8):898-909.

88. Schulz C, et al. Platelet GPVI binds to collagenous structures in the core region of human atheromatous plaque and is critical for atheroprogression in vivo. Basic Res Cardiol. 2008;103(4):356-367.

89. Jamasbi J, et al. Differential inhibition of human atherosclerotic plaque-induced platelet activation by dimeric GPVI-Fc and anti-GPVI antibodies: functional and imaging studies. J Am Coll Cardiol. 2015;65(22):2404-2415.

90. Ungerer M, et al. The GPVI-Fc fusion protein Revacept reduces thrombus formation and improves vascular dysfunction in atherosclerosis without any impact on bleeding times. PLoS ONE. 2013;8(8):e71193.

91. Busygina K, et al. Oral Bruton tyrosine kinase inhibitors selectively block atherosclerotic plaque-triggered thrombus formation in humans. Blood. 2018;131(24):2605-2616.

92. Ungerer $\mathrm{M}$, et al. Novel antiplatelet drug revacept (dimeric glycoprotein VI-Fc) specifically and efficiently inhibited collagen-induced platelet aggregation without affecting general hemostasis in humans. Circulation. 2011;123(17):1891-1899.

93. Andre P, et al. Critical role for Syk in responses to vascular injury. Blood. 2011;118(18):5000-5010.

94. Nieswandt B, Kleinschnitz C, Stoll G. Ischaemic stroke: a thrombo-inflammatory disease? JPhysiol (Lond). 2011;589(17):4115-4123.

95. Engelmann B, Massberg S. Thrombosis as an intravascular effector of innate immunity. Nat Rev Immunol. 2013;13(1):34-45.

96. Kleinschnitz C, Pozgajova M, Pham M, Bendszus M, Nieswandt B, Stoll G. Targeting platelets in acute experimental stroke: impact of glycoprotein Ib, VI, and IIb/IIIa blockade on infarct size, functional outcome, and intracranial bleeding. Circulation. 2007;115(17):2323-2330.

97. Goebel S, et al. The GPVI-Fc fusion protein Revacept improves cerebral infarct volume and functional outcome in stroke. PLOS ONE. 2013;8(7):e66960.

98. Kraft P, et al. Efficacy and safety of platelet glycoprotein receptor blockade in aged and comorbid mice with acute experimental stroke. Stroke. 2015;46(12):3502-3506.

99. Reimann A, et al. Combined administration of the GPVI-Fc fusion protein Revacept with lowdose thrombolysis in the treatment of stroke. Heart Int. 2016;11(1):e10-e16.

100.Nieswandt B, Pleines I, Bender M. Platelet adhesion and activation mechanisms in arterial thrombosis and ischaemic stroke.J Thromb Haemost. 2011;9(Suppl 1):92-104.

101.Pachel C, et al. Inhibition of platelet GPVI protects against myocardial ischemiareperfusion injury. Arterioscler Thromb Vasc Biol. 2016;36(4):629-635

102. Hitchcock JR, et al. Inflammation drives thrombosis after Salmonella infection via CLEC-2 on platelets. J Clin Invest. 2015;125(12):4429-4446.

103. Budnik I, Brill A. Immune factors in deep vein thrombosis initiation. Trends Immunol. 2018;39(8):610-623.

104.von Brühl ML, et al. Monocytes, neutrophils, and platelets cooperate to initiate and propagate venous thrombosis in mice in vivo. JExp Med. 2012;209(4):819-835.

105. Brill A, et al. Von Willebrand factor-mediated platelet adhesion is critical for deep vein thrombosis in mouse models. Blood. 2011;117(4):1400-1407.

106. Brill A, et al. Neutrophil extracellular traps promote deep vein thrombosis in mice. J Thromb 
Haemost. 2012;10(1):136-144.

107. Payne H, Ponomaryov T, Watson SP, Brill A. Mice with a deficiency in CLEC-2 are protected against deep vein thrombosis. Blood. 2017;129(14):2013-2020.

108. Müller F, et al. Platelet polyphosphates are proinflammatory and procoagulant mediators in vivo. Cell. 2009;139(6):1143-1156.

109.Xiang B, et al. Platelets protect from septic shock by inhibiting macrophage-dependent inflammation via the cyclooxygenase 1 signalling pathway. Nat Commun. 2013;4:2657.

110.Gudbrandsdottir S, Hasselbalch HC, Nielsen CH. Activated platelets enhance IL-10 secretion and reduce TNF- $\alpha$ secretion by monocytes. J Immunol. 2013;191(8):4059-4067.

111. Acton SE, et al. Dendritic cells control fibroblastic reticular network tension and lymph node expansion. Nature. 2014;514(7523):498-502.

112. de Stoppelaar SF, van 't Veer C, Claushuis TA, Albersen BJ, Roelofs JJ, van der Poll T. Thrombocytopenia impairs host defense in gramnegative pneumonia-derived sepsis in mice. Blood. 2014;124(25):3781-3790.

113. Claushuis TAM, et al. Platelet glycoprotein VI aids in local immunity during pneumoniaderived sepsis caused by gram-negative bacteria. Blood. 2018;131(8):864-876.

114. Hu H, et al. GPVI and GPIb $\alpha$ mediate staphylococcal superantigen-like protein 5 (SSL5) induced platelet activation and direct toward glycans as potential inhibitors. PLOS ONE. 2011;6(4):e19190.

115. Scopel-Guerra A, et al. The impact of helicobacter pylori urease upon platelets and consequent contributions to inflammation. Front Microbiol. 2017;8:2447.

116. Rayes J, et al. The podoplanin-CLEC-2 axis inhibits inflammation in sepsis. Nat Commun. 2017;8(1):2239.

117. Lax S, Rayes J, Thickett DR, Watson SP. Effect of anti-podoplanin antibody administration during lipopolysaccharide-induced lung injury in mice. BMJ Open Respir Res. 2017;4(1):e000257.

118. Zahn A, Jennings N, Ouwehand WH, Allain JP. Hepatitis $\mathrm{C}$ virus interacts with human platelet glycoprotein VI. J Gen Virol. 2006;87(Pt 8):2243-2251.

119. Chaipan C, et al. DC-SIGN and CLEC-2 mediate human immunodeficiency virus type 1 capture by platelets. J Virol. 2006;80(18):8951-8960.

120. Boilard E, et al. Platelets amplify inflammation in arthritis via collagen-dependent microparticle production. Science. 2010;327(5965):580-583.

121. Hsu J, Gu Y, Tan SL, Narula S, DeMartino JA, Liao C. Bruton's tyrosine linase mediates platelet receptor-induced generation of microparticles: a potential mechanism for amplification of inflammatory responses in rheumatoid arthritis synovial joints. Immunol Lett. 2013;150(1-2):97-104.

122. Devi S, et al. Platelet recruitment to the inflamed glomerulus occurs via an alphaIIbbeta3/GPVI-dependent pathway. Am J Pathol. 2010;177(3):1131-1142.

123. Pierre S, et al. GPVI and thromboxane receptor on platelets promote proinflammatory macrophage phenotypes during cutaneous inflammation. J Invest Dermatol. 2017;137(3):686-695.
124. Ekwall AK, et al. The tumour-associated glycoprotein podoplanin is expressed in fibroblast-like synoviocytes of the hyperplastic synovial lining layer in rheumatoid arthritis. Arthritis Res Ther. 2011;13(2):R40.

125. Del Rey MJ, et al. Clinicopathological correlations of podoplanin (gp38) expression in rheumatoid synovium and its potential contribution to fibroblast platelet crosstalk. PLOS ONE. 2014;9(6):e99607.

126. Croft AP, et al. Rheumatoid synovial fibroblasts differentiate into distinct subsets in the presence of cytokines and cartilage. Arthritis Res Ther. 2016;18(1):270.

127. Miyamoto Y, et al. Podoplanin is an inflammatory protein upregulated in Th17 cells in SKG arthritic joints. Mol Immunol. 2013;54(2):199-207.

128. Takakubo Y, et al. Distribution of podoplanin in synovial tissues in rheumatoid arthritis patients using biologic or conventional disease-modifying anti-rheumatic drugs. Curr Rheumatol Rev. 2017;13(1):72-78.

129. Peters A, et al. Podoplanin negatively regulates CD4+ effector T cell responses. J Clin Invest. 2015;125(1):129-140.

130. Nylander AN, et al. Podoplanin is a negative regulator of Th17 inflammation. JCI Insight. 2017;2(17):e92321.

131. Agrawal S, Ganguly S, Hajian P, Cao JN, Agrawa A. PDGF upregulates CLEC- 2 to induce T regulatory cells. Oncotarget. 2015;6(30):28621-28632.

132. Noack M, Ndongo-Thiam N, Miossec P. Interaction among activated lymphocytes and mesenchymal cells through podoplanin is critical for a high IL-17 secretion. Arthritis Res Ther. 2016;18:148.

133. Wu X, et al. Soluble CLEC2 extracellular domain improves glucose and lipid homeostasis by regulating liver Kupffer cell polarization. EBioMedicine. 2015;2(3):214-224.

134. Asai J, et al. Platelets regulate the migration of keratinocytes via podoplanin/CLEC-2 signaling during cutaneous wound healing in mice. Am J Pathol. 2016;186(1):101-108.

135. Stegner D, Dütting S, Nieswandt B. Mechanistic explanation for platelet contribution to cancer metastasis. Thromb Res. 2014; 133(Suppl 2):S149-S157.

136. Wojtukiewicz MZ, Hempel D, Sierko E, Tucker SC, Honn KV. Antiplatelet agents for cancer treatment: a real perspective or just an echo from the past? Cancer Metastasis Rev. 2017;36(2):305-329.

137. Riedl J, et al. Podoplanin expression in primary brain tumors induces platelet aggregation and increases risk of venous thromboembolism. Blood. 2017;129(13):1831-1839.

138. Jain S, Russell S, Ware J. Platelet glycoprotein VI facilitates experimental lung metastasis in syngenic mouse models. J Thromb Haemost. 2009;7(10):1713-1717.

139. Wicki A, Christofori G. The potential role of podoplanin in tumour invasion. Br J Cancer. 2007;96(1):1-5.

140.Schacht V, Dadras SS, Johnson LA, Jackson DG, Hong YK, Detmar M. Up-regulation of the lymphatic marker podoplanin, a mucin-type transmembrane glycoprotein, in human squamous cell carcinomas and germ cell tumors. Am J
Pathol. 2005;166(3):913-921.

141. Shibahara J, Kashima T, Kikuchi Y, Kunita A, Fukayama M. Podoplanin is expressed in subsets of tumors of the central nervous system. Virchows Arch. 2006;448(4):493-499.

142. Krishnan H, et al. Podoplanin: an emerging cancer biomarker and therapeutic target. Cancer Sci. 2018;109(5):1292-1299.

143. Gupta GP, Massagué J. Platelets and metastasis revisited: a novel fatty link. J Clin Invest. 2004;114(12):1691-1693.

144. Takemoto A, Miyata K, Fujita N. Plateletactivating factor podoplanin: from discovery to drug development. Cancer Metastasis Rev. 2017;36(2):225-234.

145. Shirai T, et al. C-type lectin-like receptor 2 promotes hematogenous tumor metastasis and prothrombotic state in tumor-bearing mice. J Thromb Haemost. 2017;15(3):513-525.

146. Miyata K, Takemoto A, Okumura S, Nishio M, Fujita N. Podoplanin enhances lung cancer cell growth in vivo by inducing platelet aggregation. Sci Rep. 2017;7(1):4059.

147. Sasaki T, et al. Functional characterization of recombinant snake venom rhodocytin: rhodocytin mutant blocks CLEC-2/podoplanin-dependent platelet aggregation and lung metastasis. J Thromb Haemost. 2018;16(5):960-972.

148. Shiina S, et al. CAR T cells targeting podoplanin reduce orthotopic glioblastomas in mouse brains. Cancer Immunol Res. 2016;4(3):259-268.

149. Abe S, et al. Antitumor effect of novel anti-podoplanin antibody NZ-12 against malignant pleural mesothelioma in an orthotopic xenograft model. Cancer Sci. 2016;107(9):1198-1205.

150. Chang YW, et al. Identification of a novel platelet antagonist that binds to CLEC-2 and suppresses podoplanin-induced platelet aggregation and cancer metastasis. Oncotarget. 2015;6(40):42733-42748.

151. Kato Y, Kaneko MK. A cancer-specific monoclonal antibody recognizes the aberrantly glycosylated podoplanin. Sci Rep. 2014;4:5924.

152. Critelli R, et al. Microenvironment inflammatory infiltrate drives growth speed and outcome of hepatocellular carcinoma: a prospective clinical study. Cell Death Dis. 2017;8(8):e3017.

153. Wang L, et al. C-type lectin-like receptor 2 suppresses AKT signaling and invasive activities of gastric cancer cells by blocking expression of phosphoinositide 3-kinase subunits. Gastroenterology. 2016;150(5):1183-1195.e16.

154. Hardy AT, et al. Significant hypo-responsiveness to GPVI and CLEC-2 agonists in pre-term and full-term neonatal platelets and following immune thrombocytopenia. Thromb Haemost. 2018;118(6):1009-1020.

155. Bertozzi CC, et al. Platelets regulate lymphatic vascular development through CLEC-2-SLP-76 signaling. Blood. 2010;116(4):661-670.

156. Turner M, et al. Perinatal lethality and blocked B-cell development in mice lacking the tyrosine kinase Syk. Nature. 1995;378(6554):298-302.

157. Clements JL, et al. Fetal hemorrhage and platelet dysfunction in SLP-76-deficient mice. JClin Invest. 1999;103(1):19-25.

158. Abtahian F, et al. Regulation of blood and lymphatic vascular separation by signaling proteins SLP-76 
and Syk. Science. 2003;299(5604):247-251.

159. Ichise H, Ichise T, Ohtani O, Yoshida N. Phospholipase Cgamma2 is necessary for separation of blood and lymphatic vasculature in mice. Development. 2009;136(2):191-195.

160. Carramolino L, Fuentes J, García-Andrés C, Azcoitia V, Riethmacher D, Torres M. Platelets play an essential role in separating the blood and lymphatic vasculatures during embryonic angiogenesis. Circ Res. 2010;106(7):1197-1201.

161. Stefanini L, et al. RASA3 is a critical inhibitor of RAP1-dependent platelet activation. J Clin Invest. 2015;125(4):1419-1432.

162.Hägerling R, et al. A novel multistep mechanism for initial lymphangiogenesis in mouse embryos based on ultramicroscopy. EMBO J. 2013;32(5):629-644.

163. Pollitt AY, et al. Syk and Src family kinases regulate C-type lectin receptor 2 (CLEC-2)-mediated clustering of podoplanin and platelet adhesion to lymphatic endothelial cells. J Biol Chem. 2014;289(52):35695-35710.

164. Schacht V, et al. T1alpha/podoplanin deficiency disrupts normal lymphatic vasculature formation and causes lymphedema. EMBOJ. 2003;22(14):3546-3556.

165. Ramirez MI, Millien G, Hinds A, Cao Y, Seldin DC, Williams MC. T1alpha, a lung type I cell differentiation gene, is required for normal lung cell proliferation and alveolus formation at birth. Dev Biol. 2003;256(1):61-72.

166. Millien G, Spira A, Hinds A, Wang J, Williams MC, Ramirez MI. Alterations in gene expression in T1 alpha null lung: a model of deficient alveolar sac development. BMC Dev Biol. 2006;6:35.

167. Song K, et al. Loss of mucin-type O-glycans impairs the integrity of the glomerular filtration barrier in the mouse kidney. J Biol Chem. 2017;292(40):16491-16497.

168. Bigalke B, et al. Expression of platelet glycoprotein
$\mathrm{VI}$ is associated with transient ischemic attack and stroke. Eur J Neurol.2010;17(1):111-117.

169. Bigalke B, et al. Platelet glycoprotein VI (GPVI) for early identification of acute coronary syndrome in patients with chest pain. Thromb Res. 2010;125(5):e184-e189.

170.Snoep JD, et al. The minor allele of GP6 T13254C is associated with decreased platelet activation and a reduced risk of recurrent cardiovascular events and mortality: results from the SMILE-Platelets project. J Thromb Haemost. 2010;8(11):2377-2384.

171. Mangin PH, et al. A humanized glycoprotein VI (GPVI) mouse model to assess the antithrombotic efficacies of anti-GPVI agents. J Pharmacol Exp Ther. 2012;341(1):156-163.

172. Nicolson PLR, et al. Inhibition of Btk by Btkspecific concentrations of ibrutinib acalabrutinib delays but does not block platelet aggregation to GPVI. Haematologica. 2018;103:2097-2108. 